\title{
Wind Power Prediction Based on Fuzzy Information Granulation Nan Sheng ${ }^{1}$,Guochu Chen ${ }^{1, a}$ \\ ${ }^{1}$ School of Electric Engineering,Shanghai DianJi University,Shanghai 200240,China \\ ${ }^{a}$ Corresponding author:chengc@sdju.edu.cn
}

Keywords: power forecast. wind power. fuzzy information granulation. SVM

Abstract: In this paper, a new method for predicting the value of wind power is proposed, which combine fuzzy information granulation and support vector machine.Firstly,extract the original data and make fuzzy information granulation of the original data.Secondly,do the regression prediction of the granulated data by SVM.Finally,present the data of wind power,and verify the forecasting result.

\section{Introduction}

At present, the development of wind power in China is very rapid. However, the wind energy is more easily affected by weather conditions and it has instability and randomness, which has caused some obstacles to the development of wind power. Therefore, the wind power prediction is necessary ${ }^{2}$. In this paper, fuzzy information granulation and SVM are combined, and a FIG-SVM method is proposed to predict the actual wind power. Experiments show that this method can improve the accuracy of wind power prediction, which proves the validity of this method.

\section{Support Vector Machine}

Support Vector Machine was first proposed by Vapnik. By use, SVM can be divided into support vector regression (generally used in forecasting fields) and support vector machines (generally used for classification).

\section{Support Vector Machine Regression Principle}

In order to make SVM can solve the problem of regression fitting, Vapnik, who introduced the insensitive loss function, obtained the SVR (support vector machine for regression). When SVM is used in regression fitting, the basic idea is to find an optimal classification surface, which can make the error of all the training samples be minimized to the optimal classification surface.

Establish a regression function in the high dimensional feature space.

$$
f(x)=w \varphi(x)+\delta \quad(1)
$$

Where is a nonlinear mapping function.

Define a linear insensitive loss function is $\theta$ :

$$
L(f(x), y, \theta)= \begin{cases}0, & |y-f(x)| \leq \theta \\ |y-f(x)|-\theta, & |y-f(x)|>\theta\end{cases}
$$


Whereis the predicted value of the regression function;is the corresponding true value.

Optimization problem is:

$$
\begin{aligned}
& \left(\min \frac{1}{2}\|\omega\|^{2}+K \sum_{i=1}^{n}\left(\beta_{i}+\beta_{i}^{*}\right)\right. \\
& \text { s.t. }\left\{\begin{array}{l}
y_{i}-\omega \varphi\left(x_{i}\right)-\delta \leq \theta+\beta_{i}, \\
-y_{i}+\omega \varphi\left(x_{i}\right)+\delta \leq \theta+\beta_{i}^{*} \\
\beta_{i} \geq 0, \beta_{i}^{*} \geq 0
\end{array} \quad i=1,2, \mathrm{~L} t\right.
\end{aligned}
$$

Among them,and is slack variable, $\mathrm{K}$ is penalty factor.

Introducing Lagrange function, and the optimization problem turn into:

$$
\begin{aligned}
& \left\{\begin{array}{l}
\max \left[-\frac{1}{2} \sum_{i=1}^{t} \sum_{j=1}^{t}\left(\alpha_{i}-\alpha_{i}^{*}\right)\left(\alpha_{j}-\alpha_{j}^{*}\right) \mu\left(x_{i}, x_{j}\right)-\sum_{i=1}^{t}\left(\alpha_{i}+\alpha_{i}^{*}\right) \theta+\sum_{i=1}^{t}\left(\alpha_{i}-\alpha_{i}^{*}\right) y_{i}\right] \\
\text { s.t. }\left\{\begin{array}{l}
\sum_{i=1}^{t}\left(\alpha_{i}-\alpha_{i}^{*}\right)=0 \\
0 \leq \alpha_{i} \leq K \\
0 \leq \alpha_{i}^{*} \leq K
\end{array}\right.
\end{array}\right. \\
& f(x)=\sum_{i=1}^{n}\left(\alpha_{i}-\alpha_{i}^{*}\right) k\left(x_{i} x\right)+\delta(5)
\end{aligned}
$$

\section{Fuzzy Information Granulation}

Information granulation was first proposed by Professor L.A.Zadeh. Information granules are a collection of some elements which are combined because they are approximate, or difficult to distinguish, or have some feature.

The main models of information granulation include: word computing model, rough set model, fuzzy set model, quotient space theory model ${ }^{6}$. Professor W. Pedrycz's granulation method is used in this paper $^{3}$. First of all, do the fuzzy granulation processing to the time series, and it is divided into two steps: dividing the window and fuzzy processing. Dividing the window is to divide time series into several sub sequences, which are used as the operating window; then according to the actual needs, do the fuzzy processing to each window and get every fuzzy set, which should be able to express the original window data. This is fuzzy information granulation, which is called f-granulation ${ }^{5}$.The task of fuzzy processing is to set up a fuzzy granule $\mathrm{K}$, which should be able to reasonably describe the fuzzy set and the set regard $\mathrm{X}$ as domain. The conditions of establishing fuzzy granule $\mathrm{K}$ are: ( 1 ) $\mathrm{K}$ should reasonably represent the original data ( 2 ) K must have some special characteristics. In order to meet these two conditions and to find the best balance between the two, establishing a function can be considered:

$Q_{K}=\frac{M_{K}}{N_{K}}(6)$

Among them, satisfies the condition (1), satisfies the condition (2).

There are several basic forms of fuzzy granule: trapezoidal, triangular, parabolic, Gauss and so 
on. Triangular form is used in this paper, and the membership functions are as follows:

$$
\mu(x, \alpha, \beta, \gamma)=\left\{\begin{array}{l}
0, x<\alpha \cup x>\gamma \\
\frac{x-\alpha}{\beta-\gamma}, \alpha \leq x \leq \beta \quad(7) \\
\frac{\gamma-x}{\gamma-\beta}, \beta<x \leq \gamma
\end{array}\right.
$$

Among them, $\mathrm{x}$ is the variable in the domain and are parameters. For triangular function, Low represents the minimum value of the original data change, $\mathrm{R}$ represents the average value of the original data change, and up represents the maximum value of the original data change.

\section{Wind Power Prediction That Information Granulation And SVM Combined}

In this paper, information granulation and SVM is combined to predict the wind power. Specific procedures are shown in Figure 1, the concrete steps are as follows:

1 ) Firstly, extract the data and determine the window. Divide the time series into several sub sequences and do fuzzy information granulation to the original data.

2 )Get Low(the minimum value of the original data change), $R$ (the average value of the original data change), and $U p$ (the maximum value of the original data change) after information granulation. Make preprocessing for the data and normalized processing for Low, R, Up. The normalized formula is shown in formula (11)

$$
x_{t}=\frac{p_{t}-p_{\min }}{p_{\text {max }}-p_{\text {min }}}(8)
$$

3 ) After establishing support vector machine model by using the normalized data, select the optimal SVM parameters c\&g and put the best c\&g into the regression function(5) to get the wind power prediction model.

4 ) Predict Low, R and Up by the prediction model.

\section{5 ) Analyze the forecast results}

In this paper mean squared error and squared correlation coefficient are used to analyze the prediction results.

\section{Practical Application}

Based on the data of 30 days in July, 2010, a wind farm in Northeast China was sampled, and a total of 720 sets of data were sampled. Do preprocessing to all power data by fuzzy information granulation and the granulated data is shown in Figure 1.Among them, Low represents the minimum value of the original data change, $\mathrm{R}$ represents the average value of the original data change, and up represents the maximum value of the original data change. 


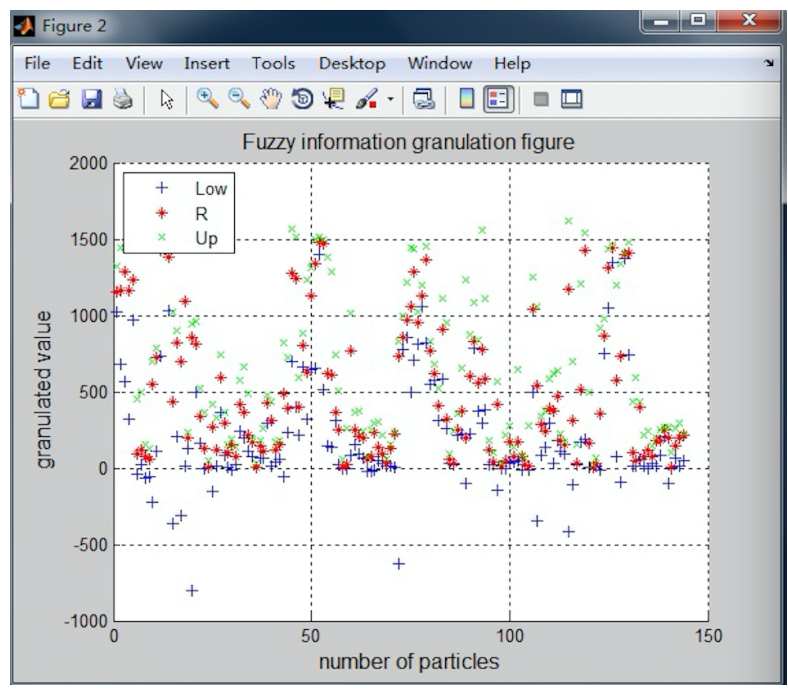

Fig.1 Fuzzy information granulation figure

According to the formula ( 11 ), do normalization processing to Low. The normalized image of Low is shown in figure 2.

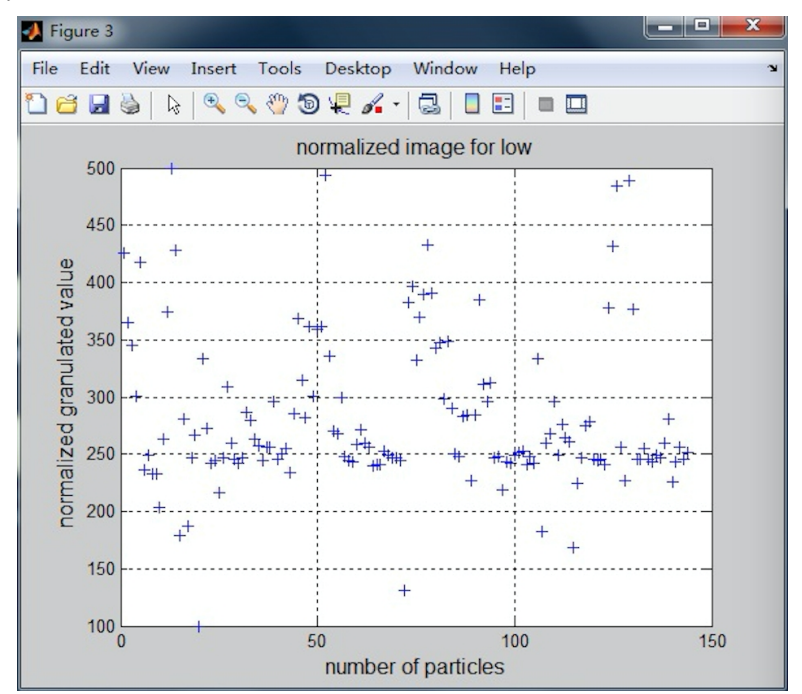

Fig.2 Normalized image of Low

Similarly, do the normalization processing to $\mathrm{R}$ and Up, and establish the model of support vector machine by normalized Low, $\mathrm{R}$ and Up. Select the optimal parameters of SVM to obtain the prediction model of wind power. Using the forecasting model to predict Low, $\mathrm{R}$ and Up. Using mean squared error and squared correlation coefficient to do error analysis for the prediction results. The mean squared error and squared correlation coefficient of the SVM model(SVM)and the fuzzy information granulated SVM model(FIG-SVM) are shown in Table 1.

Table 1

\begin{tabular}{|c|c|c|}
\hline & SVM & FIG-SVM \\
\hline Mean Squared Error & 0.0487277 & 0.0416244 \\
\hline Squared Correlation Coefficient & 0.876211 & 0.882943 \\
\hline
\end{tabular}

As we can see from Table 1,compared with SVM, the mean squared error of fuzzy information granulated SVM model(FIG-SVM) is smaller, and the squared correlation coefficient is more close 
to 1. So the FIG-SVM is more accurate.

\section{Conclusion}

In this paper, fuzzy information granulation and support vector machine are combined to predict the wind power. The experimental results show that FIG-SVM is effective for the wind power prediction, and the prediction accuracy is improved. It provides a theoretical basis for the smooth operation of the wind farm.

\section{Acknowledgements}

This work was financially supported by scientific research innovation projects of Shanghai municipal education commission(Grant No.13YZ140) and the key disciplines of Shanghai Municipal Education Commission of China(Grant No.J51901).

\section{References}

[1].BARGIELA,PEDRYCZ W. Granular computing:an introduction. Dodrecht: Kluwer Academic Publishers(2003)

[2]. Dong Lei,Liaozhong, Wang Lijie (2014) Modeling and prediction of wind power generation in the large wind farm(2014): 39-59

[3].WitoldPedrycz. Knowledge-Based Clustering: From Data to Information Granules. Beijing Normal University press(2008):3-73

[4].XuJiucheng,Sun Lin,Zhang Qianqian. The theory and method of granular computing and its uncertain information measurement. Beijing: Science Press:24-49

[5].WitoldPedrycz. Principles and methodology of fuzzy sets. Journal of Intelligent Manufacturing ( 1993$)$

[6]. Zhang Ling,Zhang Bo. Theory of fuzzy quotient space(methods of fuzzy granular computing).Journal of Software.(2013):770-776

[7]. Ye Lin,Liu Peng. Combined Model Based on EMD-SVM for Short-term Wind Power Prediction. Proceedings of the CSEE ( 2011 ) 